\title{
HER-2/neu overexpression correlates with increased expression of VEGF in primary breast carcinoma
}

\author{
Sahu $\mathbf{N}^{1}$, Sharma $\mathbf{A}^{2}$, Senapati $\mathbf{U}^{3}$ \\ ${ }^{1}$ Dr Nageswar Sahu, Pathology, Assistant Professor, Department of Pathology, Kalinga Institute of Medical Sciences \\ (KIMS), Bhubaneswar, Odisha, India, ${ }^{2}$ Dr Anurag Sharma, Pathology, Assistant Professor, Department of Pathology, \\ Government Medical College, Rajnandgaon, Chhattisgarh, India, ${ }^{3}$ Dr Urmila Senapati, Professor \& HOD, Department of \\ Pathology, Kalinga Institute of Medical Sciences (KIMS), Bhubaneswar, Odisha, India.
}

Address for Correspondence: Dr Nageswar Sahu, Email: nageswar.sahu@yahoo.in

\begin{abstract}
Background: Overexpression of HER-2/neu is associated with up-regulation of vascular endothelial growth factor (VEGF) in human breast cancer cells in vitro. Preclinical experiments indicate that increased expression of VEGF may in part mediate the biologically aggressive phenotype of HER-2/neu overexpressing human breast cancer. It was the purpose of this study to: (a) evaluate the association between HER-2/neu and VEGF expression in a cohort of primary breast cancer patients; (b) find out any association of HER-2/neu or VEGF with traditional histologic prognostic parameters. Materials and Methods: HER-2/neu and VEGF were measured by immunohistochemistry in 70 cases of newly diagnosed primary breast cancers and the relation between these two markers and their relations with traditional prognostic factors were analyzed by statistical methods. Results: Our findings indicate a significant positive association between HER-2/neu and VEGF expression. VEGF did not show significant association with tumor size, histologic grade or lymph node status. HER-2/neu overexpression was maximum in grade II, followed by grade I and III with a significant $\mathrm{p}$ value $(<0.001)$. But HER-2/neu didn't have any association with tumor size or lymph node status. Conclusion: The positive association between HER-2/neu and VEGF expression implicates VEGF in the aggressive phenotype exhibited by HER-2/neu overexpression, and supports the use of combination therapies directed against both HER-2/neu and VEGF for treatment of breast cancers that overexpress HER-2/neu. The unusual association found between HER-2/neu and grade of tumor may be due to uneven distribution of cases among different grades of tumor.
\end{abstract}

Keywords: Breast carcinoma, HER-2/neu, Prognostic factors, VEGF

\section{Introduction}

Breast cancer is by far the most common cancer diagnosed in women in the world. The incidence of breast cancer is rising in India and is now the second most commonly diagnosed cancer in women after cervical cancer [1]. Although invasive carcinoma of breast was clinically regarded as a single entity in the past, histologic and molecular analysis have demonstrated that breast cancer is a heterogeneous disease, composed of morphologically and genetically distinct entities with different molecular profiles, behavior, and response to therapy [2]. The HER-2/neu is an epidermal growth factor receptor. HER-2/neu

Manuscript received $8^{\text {th }}$ November 2016

Reviewed: $20^{\text {th }}$ November 2016

Author Corrected: $4^{\text {th }}$ December 2016

Accepted for Publication 17 $7^{\text {th }}$ December 2016 proto-oncogene transcribes the HER-2/neu protein which is a tyrosine kinase transmembrane receptor protein. HER2/neu over expression has been reported to be associated with positive lymph nodes, high histologic grade, high proliferation rate and lack of expression of estrogen and progesterone receptors in breast cancer. It is also associated with a more aggressive phenotype with decreased survival rate [3].

Angiogenesis is an important step in the development of cancer and is necessary for primary tumor growth, invasiveness and metastasis. Multiple angiogenic factors are commonly expressed by invasive human breast cancers: the vascular endothelial growth factor (VEGF or VEGF-A) being predominant. The role of VEGF in 
breast cancer is not limited to angiogenesis. Like endothelial cells, breast cancer cells themselves express both VEGF and VEGF receptors (VEGFRs) [4,5]. VEGF-A interacts with its specific receptors VEGFR-1 and VEGFR-2. VEGFR-2 is functional on the surface of breast cancer cells. These cancer cell receptors are capable of being stimulated by VEGF, indicating the presence of an autocrine signaling loop distinct from the angiogenic effects of VEGF [6,7]. Thus, breast cancer cells may be able to promote their own growth and avoid apoptosis through VEGF [4]. Higher levels of VEGF protein have been shown to correlate with poor prognosis in breast cancer patients, irrespective of lymph node status $[8,9]$. In addition, the possibility that VEGF predicts response to certain types of therapy has also been explored. The relationship between increased VEGF expression and poor response to conventional systemic therapy, specifically tamoxifen or chemotherapy has been demonstrated [10].

Numerous studies implicate the fact that activated HER2/neu can potentiate tumor cell adhesion to endothelial cells, increase VEGF expression and facilitate angiogenesis and vascular invasion [11,12,13]. A statistically significant association between immunehistochemical expression of HER-2/neu and VEGF was first demonstrated by Wentao Yang et al in 2002[14]. After that many studies demonstrated a significant positive association between HER-2/neu and VEGF in breast carcinoma $[15,16,17]$. Specific molecular targeted therapy named herceptin is available to treat patients with an overexpression of HER-2/neu [3]. The strong association between HER-2/neu and VEGF in breast cancer has led to a change in treatment strategy with patients having overexpression of HER-2/neu. Newer modalities of treatment like combination therapy of anti-HER-2/neu with anti-VEGF or combination of HER-2/neu and VEGF peptide mimics are now a days implemented in many clinical trial[18,19,20].

An association between HER-2/neu overexpression and VEGF expression could strengthen the hypothesis based on preclinical evidence that increased VEGF expression may be a result of HER-2/neu overexpression and may contribute to the aggressive phenotype of HER-2/neu overexpressing breast cancer cells. This would provide a rationale for combined therapeutic approaches targeting both VEGF and HER-2/neu. The primary aim of the present study was to determine possible associations between expressions of HER-2/neu and VEGF in breast carcinoma. A secondary aim was to see the association of HER-2/neu and VEGF with traditional prognostic factors like tumor size, grade and axillary lymph node status.

\section{Materials and Methods}

The present study was done in our Department of Pathology with approval from institutional ethics committee. It included 70 cases of primary carcinoma breast, confirmed by biopsy. Only cases undergoing complete tumor excision with axillary clearance were included. Cases with neoadjuvant chemotherapy or radiotherapy were excluded. Also cases having equivocal HER-2/neu score (score-2) which requires further test like ISH (In Situ Hybridization) were not included.

Immunohistochemical analysis- The antibodies \& chemicals were obtained from Biogenex, USA. The anti-VEGF (clone 165) and anti human C-erb-B2 (clone CB11) monoclonal antibodies were used for VEGF and HER-2/neu respectively. Immunohistochemical evaluation was done on formalin fixed paraffin embedded tissue sections on poly-L lysine coated slides by using polymer two step indirect method. The manufacturer's instructions were followed after standardization in our laboratory. Deparaffinization was done by placing slides on hot plates at $60^{\circ} \mathrm{C}$ for 30 minutes followed by xylene. This was followed by rehydration (in decreasing concentration of alcohol) and washing in running tap water and deiodinized water. Antigen retrieval (Microwave heating method) was done by dipping the slides in antigen retrieval solution at $\mathrm{pH}-9$ and heated in the microwave oven. After cooling and washing in wash buffer solution, peroxide block solution was added to block endogenous peroxidase. Then power block solution was added to prevent background staining. The slides were incubated with primary antibody for 60 minutes at room temperature followed by washing in wash buffer solution. Then slides were incubated with secondary antibody tagged with HRP (horseradish protein) for 30 minutes at room temperature followed by washing in wash buffer solution and addition of DAB (diaminobenzidine) solution. The slides were washed in distilled water and counter stained with hematoxylin followed by dehydration (in increasing concentrations of alcohol) and mounting.

External positive and negative control slides were used with each batch of staining. Positive control slides were prepared from breast carcinoma (for HER-2/neu) and 
adenocarcinoma colon (for VEGF) known to be positive for them. Negative control slides were prepared from the same tumor block under study, but the primary antibody step was omitted and slides were incubated with PBS (phosphate buffer saline) instead.

For HER-2/neu, the newer (2013) ASCO/CAP guidelines was used [21].

- 0 (Negative) - No staining observed or membrane staining that is incomplete and is faint/barely perceptible and within $\leq 10 \%$ of the invasive tumor cells

- 1+ (Negative) - Incomplete membrane staining that is faint/barely perceptible and within $>10 \%$ of the invasive tumor cells

- 2+ (equivocal)- Circumferential membrane staining that is incomplete and/or weak/moderate and within $>10 \%$ of the invasive tumor cells; or complete and circumferential membrane staining that is intense and within $\leq 10 \%$ of the invasive tumor cells

- 3+ (Positive) - Circumferential membrane staining that is complete, intense and within $>10 \%$ of the invasive tumor cells.
Score 2 (equivocal) requires further test e.g. ISH for confirmation and were excluded from the study. For VEGF, cytoplasmic staining was evaluated with reference to both the staining intensity and the percentage of positive cells [22]. Staining intensity was scored as: Negative-0, Weak-1, Medium-2 and Strong3. Percentage positivity of cells was scored as: nil-0, 1$25 \%$ as $1,26-50 \%$ as $2,51-75 \%$ as 3 and $76-100 \%$ as 4 . The sum of both the above scores was used as final score (0-7). Tumors having a final staining score of $\geq 3$ were considered to be positive.

The assessment of immunohistochemical staining was carried out by two independent pathologists. In one case final score for VEGF differed between the two observers and that case was reinvestigated by both investigators on a multiheaded microscope.

Statistical analysis: The data analysis was done using SPSS software. The Pearson Chi-square test was used to investigate associations between all the variables. $\mathrm{P}$ value $\leq 0.05$ was set as the significance level.

\section{Results}

To investigate the association between HER-2/neu overexpression and VEGF expression these two markers were studied in a cohort of 70 cases of primary breast cancer patients. Both the markers were studied by immunohistochemistry. Table-1 shows the distribution of cases in relation to age, tumor size, histologic grade, axillary lymph node status and HER-2/neu and VEGF positivity.

Table-1: Distribution of cases in relation to clinicopathological parameters

\begin{tabular}{|c|c|c|c|}
\hline \multicolumn{2}{|c|}{ Parameters } & Number & Percentage \\
\hline Age(years) & $\leq 50$ & 48 & 38.60 \\
\cline { 2 - 4 } & $>50$ & 22 & 5.80 \\
\hline \multirow{3}{*}{ Tumor size } & T1(<2cm) & 04 & 67.10 \\
\cline { 2 - 4 } & $\mathrm{T} 2(2-5 \mathrm{~cm})$ & 47 & 27.10 \\
\cline { 2 - 4 } & $\mathrm{T} 3(>5 \mathrm{~cm})$ & 19 & 11.40 \\
\hline \multirow{3}{*}{ Histologic grade } & I & 08 & 47.10 \\
\cline { 2 - 4 } & II & 33 & 41.40 \\
\cline { 2 - 4 } & III & 29 & 62.90 \\
\hline Lymphnode metastasis & Present & 44 & 37.10 \\
\cline { 2 - 4 } & Absent & 26 & 50.00 \\
\hline HER-2/neu & Positive & 35 & 50.00 \\
\cline { 2 - 4 } & Negative & 35 & 71.40 \\
\hline
\end{tabular}

The age group ranges from 30 year to 68 year with a mean age of 47.5 year. All the patients were female. Two third of cases were T2 lesions (2-5cm). Most of the cases belong to grade II or III, while grade I constitute only $11.40 \%$ of cases. 
Axillary lymph node metastasis was seen in $63 \%$ of cases. Half of the patients had HER-2/neu overexpressing tumor while VEGF expression was seen in $>70 \%$ of cases.

Association of HER-2/neu and VEGF with different histological parameters: Before analyzing the possible association between HER-2/neu and VEGF we tried to find out any association of these two markers with different histological characteristics. HER-2/neu overexpression was associated with histologic grade but not with tumor size and lymph node involvement, while VEGF expression was not associated with any of the parameters (Table-2). HER-2/neu overexpression was observed in 50\%, $75.80 \%$ and $20.70 \%$ cases among histologic grades I, II and III respectively. This indicated higher expression of HER-2/neu in grade II followed by grade I and least in grade III. This finding of lower HER-2/neu positivity in the worst histologic grade is unusual.

Table-2: Association of HER-2/neu and VEGF with different histological parameters.

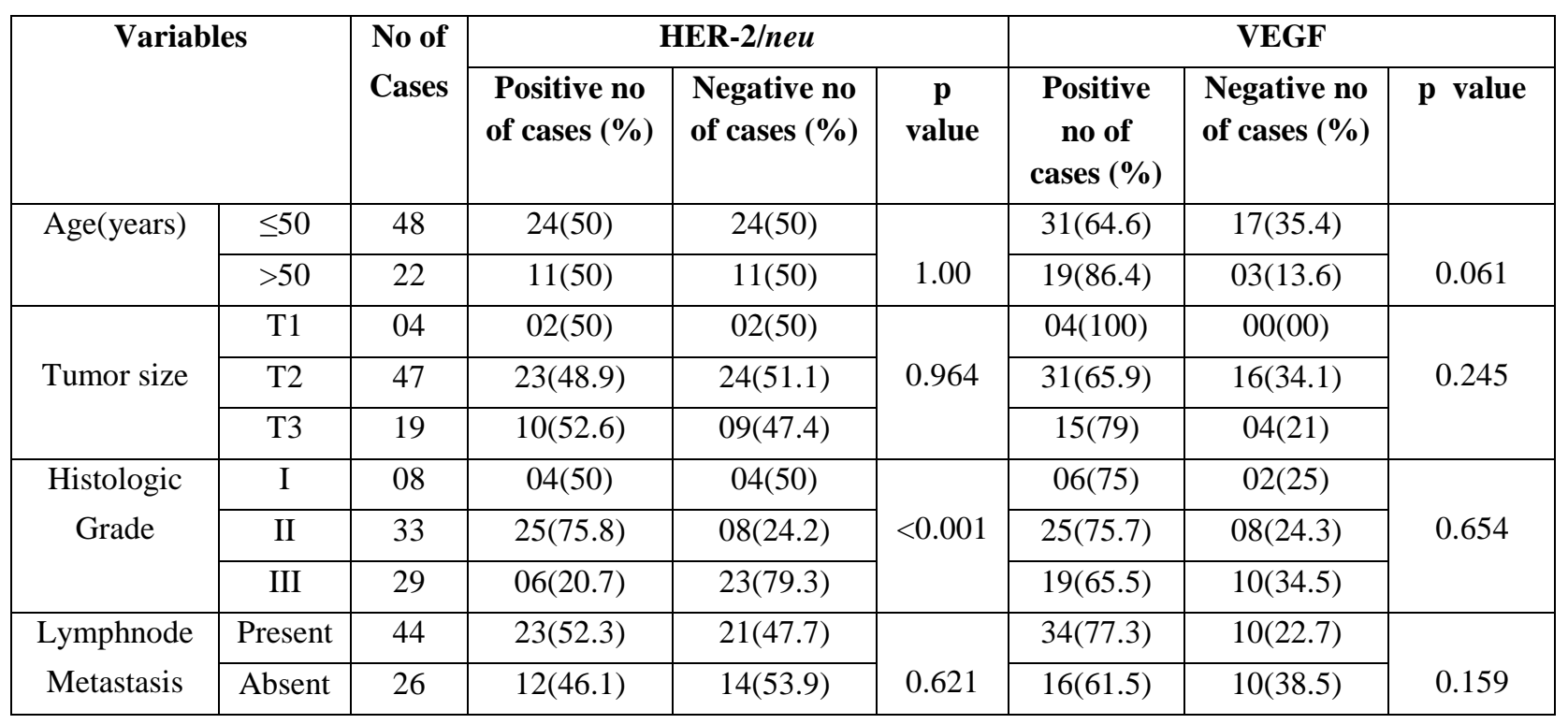

Association between HER-2/neu and VEGF: The primary objective of this study was to find the association between HER-2/neu and VEGF expression in breast carcinoma. Out of 70 cases under study, 35 were HER-2/neu positive and 35 were HER-2/neu negative showing an even distribution. VEGF expression was seen in all (35 of 35) patients with HER2/neu overexpressing tumors in contrast to 15 of 35 (42.80\%) of patients with HER-2/neu non-overexpressing tumors $(\mathrm{p}<0.001)$ confirming a positive association between HER-2/neu overexpression and VEGF expression (Table-3).

Table-3: Association between HER-2/neu and VEGF.

\begin{tabular}{|c|c|c|c|c|}
\hline \multirow{2}{*}{ HER2/neu } & \multirow{2}{*}{ No of cases } & \multicolumn{2}{|c|}{ VEGF } & p value \\
\cline { 2 - 4 } & & Positive (\%) & Negative (\%) & $<0.001$ \\
\hline Positive & 35 & $35(100)$ & $00(00)$ & \\
\hline Negative & 35 & $15(42.8)$ & $20(57.2)$ & \\
\hline
\end{tabular}

\section{Discussion}

The development of breast cancer involves progression through a series of intermediate processes, starting with ductal hyperproliferation, followed by subsequent evolution to carcinoma in situ, invasive carcinoma and finally into metastatic disease. Given the variability in clinical progression of the disease, the identification of markers could be useful to predict tumor behavior, for diagnosis and prediction of prognosis, as well as in the development of new treatment modalities. The status of estrogen receptor (ER), progesterone receptor (PR), and human epidermal growth factor receptor type 2 (HER2) has been used as predictive markers for identifying a high-risk phenotype and for selection of the most efficient therapies [23]. 
HER-2/neu overexpression is associated with increased tumor progression and metastasis; however, the exact mechanisms by which HER-2/neu regulates this more aggressive clinical phenotype are not fully understood. Recent studies indicate that HER-2/neu receptors play an important role in heregulin-induced angiogenesis. Overexpression of the HER-2/neu receptor alone results in induction of the basal level of VEGF, and exposure to heregulin $\beta 1$ additionally enhances VEGF secretion in breast cancer cells. These experimental data indicate that an important consequence of HER-2/neu signaling is increased VEGF expression. VEGF in turn is a central regulator of angiogenesis, suggesting that the aggressive phenotype of HER-2/neu overexpressing breast cancers may be in part attributable to increased angiogenesis [15].

Based on the strong positive association between HER$2 /$ neu and VEGF in breast carcinoma two newer effective modalities of treatment have been proposed. Numerous studies have been conducted to find out the efficacy of combined therapy directed against both HER-2/neu and VEGF. Conflicting data exist regarding this. In the BETH trial, patients were randomly assigned to receive chemotherapy plus trastuzumab (anti-HER2/neu) plus bevacizumab (anti-VEGF) or chemotherapy and trastuzumab alone. No difference of efficacy was observed between the two groups [19]. On the other hand, few studies showed that the combined double administration of bevacizumab and trastuzumab was clinically effective, safe and non-toxic [20]. However according to Foy et al[18] there are many drawbacks of the above modality of treatment like too expensive, limited duration of action, development of drug resistance and serious side effects. In fact combination treatment with HER-2/neu and VEGF peptide mimics induces potent antitumor and antiagiogenic effect as immunotherapeutic strategies. This shows that targeting the two different receptors (whether antagonist or agonists) will produce greater anti-tumor and antiangiogenic effects both in vitro and in vivo [18].

Our study was designed with the primary objective of finding an association between immunohistochemical expressions of HER-2/neu and VEGF in breast cancer patients. The study of this association was important because VEGF has been implicated in aggressive phenotype of breast cancers that overexpress HER$2 / n e u$. The reaffirmation of this association would support the use of combination therapies targeting both HER-2/neu and VEGF for treatment of breast cancers that show HER-2/neu overexpression. The secondary objectives were to correlate the immunohistochemical expressions of HER-2/neu and VEGF with various pathologic parameters.

Tumor size, histologic grade and lymph node status are the traditional prognostic factors in patients with invasive breast cancer [24]. In our study VEGF did not show significant association with any of these three parameters, but HER-2/neu had a non-linear association with grade $(p<0.001)$. Variable results seen in recent studies regarding the relationship of these two markers with these prognostic factors. Xiaowei Ye et al[25] in a study of 117 cases of primary breast cancer found no significant association of HER-2/neu or VEGF with tumor size, but Konecny et al[15] in their study of 611 cases of breast cancer found a positive correlation between VEGF and tumor size. In a study of 286 cases of primary breast cancer Qiao et al[17] found a positive association between HER-2/neu and grade, and Konecny et al demonstrated a positive correlation between grade and VEGF expression. The finding of lower HER-2/neu positivity in grade III than grade II and I tumors, as seen in the present study was also observed in a study of small series of 30 cases of primary breast cancer by Esraa A. AL-Dujaily et al[26].

This unusual finding in our study could be attributed to the small sample size and unequal proportions of tumors among grade I, II and III. Konecny et al found no association between VEGF and nodal status, but Xiaowei Ye et al demonstrated a positive correlation between HER2/neu and VEGF with lymph node status $(\mathrm{p}=0.0418)$.

In the present study, a significant association between HER-2/neu overexpression and VEGF expression was demonstrated, with all patients having HER-2/neu overexpressing tumors demonstrating VEGF expression, as opposed to $42.8 \%$ of their HER-2/neu nonoverexpressing counterparts $(\mathrm{P}<0.001)$. These clinical data strengthens the preclinical experimental evidence indicating that HER-2/neu signaling can enhance the ability of a tumor to recruit a neovascular blood supply after the induction of VEGF expression.

The molecular basis for the association between HER2/neu overexpression and VEGF expression is explained in recent studies. VEGF gene transcription can be mediated by hypoxia inducible factor (HIF-1), which is a heterodimeric transcription factor [12,27]. 
HIF-1 activity is increased by both intratumoral hypoxia and genetic alterations, including loss of function mutations in tumor suppressor genes, as well as gain of functional alterations in oncogenes that activate the mitogen-activated protein kinase (MAPK) signal transduction pathways and the phosphatidylinositol 3'kinase (PI3K) pathway [15]. Under physiological condition HIF-1 is degraded by the ubiquitin pathway; however, under hypoxic conditions, HIF-1 is stabilized, and expression is increased as a result of decreased ubiquination. In contrast, HER-2/neu signaling induces HIF-1 protein synthesis rather than inhibiting its degradation [12]. Both mechanisms, hypoxia-driven inhibited degradation and HER-2/neu-stimulated increased synthesis of HIF-1, can therefore independently contribute to the up-regulation of VEGF [12,27].

To date, most of the clinical data regarding the association between HER-2/neu overexpression and VEGF expression in breast cancer, demonstrate a positive association between these two factors among large series of primary breast cancer patients $[14,15,16,17,25]$. Kimberly et al [28] in a study of 425 patients with metastatic breast cancer demonstrated a significant positive correlation between HER-2/neu amplification and mean microvessel density (MVD). However, in contrast to most of the studies, they found significant lower levels of VEGF expression in HER2/neu amplified tumors. The authors attributed this finding to the fact that higher amount of angiogenesis in HER-2/neu amplified tumors may lead to less tumor hypoxia and in turn decreased production of VEGF. Alternatively, they also suggested that this finding may be explained by complexity of VEGF regulation. The current study in primary breast cancer patients, however, clearly supports the observation that HER$2 /$ neu overexpression is associated with VEGF expression.

The circumstance that VEGF was not detectable in $28.60 \%$ of the samples in our study could be explained by the possibility that VEGF is not the only proangiogenic factor expressed in human breast cancers. Platelet-derived endothelial growth factor, placenta growth factor, acidic and basic fibroblast growth factor, transforming growth factor $\alpha$ and $\beta$, hepatocyte growth factor, and pleiotrophin have also been shown to be present during breast cancer progression, and any single or combination of these molecules is capable of inducing angiogenesis[15].

\section{Conclusion}

The current study provides clinical evidence that HER$2 /$ neu overexpression is associated with expression of VEGF in breast cancer, suggesting that VEGF may in part mediate the aggressive phenotype of breast cancers that overexpress HER-2/neu.

These data additionally support the use of combination therapies directed against both HER-2/neu and VEGF for treatment of breast cancers that exhibit HER-2/neu overexpression. However, this study included small number of cases, so further studies with larger sample size are required in this area to conclude an unequivocal association between HER-2/neu and VEGF in breast cancer and its implication in guiding therapy against HER-2/neu overexpressing tumors.

Funding: Nil, Conflict of interest: None initiated, Permission from IRB: Yes

\section{References}

1. Salceda S, Caro J. Hypoxia-inducible factor 1alpha (HIF-1alpha) protein is rapidly degraded by the ubiquitin-proteasome system under normoxic conditions. Its stabilization by hypoxia depends on redox-induced changes. J Biol Chem.1997 Sep 5;272 (36): 22642-47.

2. Rosai J. Breast. In: Chan JKC, Arber DA, Brunning RD, Desmet VJ, Demasters BKK, Ordonez NG, Rosenblum MK, Tallini G, editors. Rosai and Ackerman's Surgical Pathology. 10th ed. St. Louis: Elsevier; 2011.p1659-1770

3. Makroo RN, Chowdhry M, Kumar M, Srivastava P, Tyagi R, Bhadauria P, Kaul S, Sarin R, Das PK, Dua H. Correlation between HER2 gene amplification and protein overexpression through fluorescence in situ hybridization and immunohistochemistry in breast carcinoma patients. Indian J Pathol Microbiol. 2012 Oct-Dec;55(4):481-84. Doi:10.4103/0377-4929.107785.

4. Xie K, Wei D, Shi Q, Huang S. Constitutive and inducible expression and regulation of vascular endothelial growth factor. Cytokine \& Growth Factor Reviews. 2004;15(5): 297-324. DOI 10.1016/j. cytogfr. 2004.04.003

5. Schneider BP, Miller KD. Angiogenesis of Breast Cancer. J Clin Oncol. 2005 March 10; 23(8):1782-90. 
6. Maae, E, Nielsen M, Steffensen KD, Jakobsen EH, Jakobsen A, Sørensen FB. Estimation of Immunohistochemical Expression of VEGF in Ductal Carcinomas of the Breast. J Histochem Cytochem. 2011 Aug; 59(8):750 -760. doi: 10.1369/0022155411412599

7. Weigand M, Hantel P, Kreienberg R, Waltenberger J. Autocrine vascular endothelial growth factor signaling in breast cancer: Evidence from cell lines and primary breast cancer cultures in vitro. Angiogenesis. 2005;8 (3):197-204. Epub 2005 Nov 19.

8. Linderholm B, Grankvist K, Wilking N, Johansson M, Tavelin B, Henriksson R. Correlation of Vascular Endothelial Growth Factor Content With Recurrences, Survival, and First Relapse Site in Primary Nodepositive Breast Carcinoma After Adjuvant Treatment. J Clin Oncol. 2000 (April) 18(7): 1423-31.

9. Gasparini G, Toi M, Miceli R, Vermeulen PB, Dittadi R, Biganzoli E, Morabito A, Fanelli M, Gatti C, Suzuki H, Tominaga T, Dirix LY, Gion M. Clinical relevance of vascular endothelial growth factor and thymidine phosphorylase in patients with node-positive breast cancer treated with either adjuvant chemotherapy or hormone therapy. Cancer J Sci Am. 1999 Mar-Apr; 5(2):101-11.

10. Foekens JA, Peters HA, Grebenchtchikov N, Look MP, Meijer-van Gelder ME, Geurts-Moespot A, van der Kwast TH, Sweep CG, Klijn JG. High tumor levels of vascular endothelial growth factor predict poor response to systemic therapy in advanced breast cancer. Cancer Res. 2001 Jul 15;61(14):5407-14.

11. Petit AM, Rak J, Hung MC, Rockwell P, Goldstein N, Fendly B, Kerbel RS. Neutralizing antibodies against epidermal growth factor and ErbB-2/neu receptor tyrosine kinases down-regulate vascular endothelial growth factor production by tumor cells in vitro and in vivo: angiogenic implications for signal transduction therapy of solid tumors. Am J Pathol. 1997 Dec; 151(6):1523-30.

12. Laughner E, Taghavi $\mathrm{P}$, Chiles $\mathrm{K}$, Mahon PC, Semenza GL. HER2 (neu) Signaling Increases the Rate of Hypoxia-Inducible Factor $1 \alpha$ (HIF-1 $\alpha$ ) Synthesis: Novel Mechanism for HIF-1 Mediated Vascular Endothelial Growth Factor Expression. Mol Cell Biol. 2001(June); 21(12):3995-4004. DOI: 10.1128/MCB.21. 12. 3995-4004.2001.
13. Xiong S, Grijalva R, Zhang L, Nguyen NT, Pisters $\mathrm{PW}$, Pollock RE, Yu D. Up regulation of vascular endothelial growth factor in breast cancer cells by heregulin-beta1-activated P38 signaling pathway enhances endothelial cell migration. Cancer Res. 2001 Feb 15;61(4):1727-32.

14. Yang W, Klos K, Yang Y, Smith TL, Shi D, Yu D. ErbB2 over expression correlates with increased expression of vascular endothelial growth factors A, C, and D in human breast carcinoma. Cancer. 2002 Jun 1; 94(11):2855-61.

15. Konecny GE, Meng YG, Untch M, Wang HJ, Bauerfeind I, Epstein M, Stieber P, Vernes JM, Gutierrez J, Hong K, Beryt M, Hepp H, Slamon DJ, Pegram MD. Association between HER-2/neu and Vascular Endothelial Growth Factor Expression Predicts Clinical Outcome in Primary Breast Cancer Patients. Clin Cancer Res. 2004 March 1; 10(5): 1706-16.

16. Linderholm B, Andersson J, Lindh B, Beckman L, Erlanson M, Edin K, Tavelin B, Grankvist K, Henriksson R. Overexpression of c-erbB-2 is related to a higher expression of vascular endothelial growth factor (VEGF) and constitutes an independent prognostic factor in primary node-positive breast cancer after adjuvant systemic treatment. Eur J Cancer. 2004 Jan; 40(1):33-42.

17. Qiao EQ, Ji M, Wu J, Li J, Xu X, Ma R, Zhang X, He Y, Zha Q, Song X, Zhu L, Tang JH. Joint detection of multiple immunohistochemical indices and clinical significance in breast cancer. Mol Clin Oncol. 2013 Jul;1(4):703-10. Epub 2013 May 8.

18. Foy KC, Liu Z, Phillips G, Miller M, Kaumaya PT. Combination Treatment with HER-2 and VEGF Peptide Mimics Induces Potent Anti-tumor and Anti-angiogenic Responses in Vitro and in Vivo. J Biol Chem. 2011 Apr 15; 286 (15):13626-37. doi: 10.1074/jbc.M110.216820. Epub 2011 Feb 16.

19. Baker H: 2013 San Antonio Breast Cancer Symposium. Lancet Oncol. 2014 Feb; 15(2):138.

20. Königsberg R, Maierhofer J, Steininger T, Kienzer G, Dittrich C. Long-term remission of a Her2/neu positive primary breast cancer under double monoclonal antibody therapy with trastuzumab and bevacizumab. 
Radiol Oncol. 2014 Apr 25;48(2):184-88. doi: 10.2478/ raon-2013-0083.

21. Wolff AC, Hammond ME, Hicks DG, Dowsett M, McShane LM, Allison KH, Allred DC, Bartlett JMS, Bilous M, Fitzgibbons P, Hanna W, Jenkins RB, Mangu PB, Paik S, Perez EA, Press MF, Spears PA, Vance GH, Viale G, Hayes DF, American Society of Clinical Oncology, College of American Pathologists. Recommendations for human epidermal growth factor receptor 2 testing in breast cancer: American Society of Clinical Oncology/College of American Pathologists clinical practice guideline update. J Clin Oncol. 2013 Nov 1; 31(31):3997-4013. doi: 10.1200/JCO.2013.50. 9984. Epub 2013 Oct 7.A

22. El-Gendi S, Abdel-Hadi M. Lymphatic vessel density as prognostic factor in breast carcinoma: relation to clinicopathologic parameters. J Egypt Natl Canc Inst. 2009 Jun;21(2):139-49.

23. Hirata BKB, Oda JMM, Guembarovski RL, Ariza CB, Coral de Oliveira CE, Watanabe MAE. Molecular Markers for Breast Cancer: Prediction on Tumor Behavior. Hindawi Publishing Corporation. Hindawi Publishing Corporation. Disease Markers Volume 2014, Article ID 513158, 12 pages http://dx.doi. org/10. $1155 / 2014 / 513158$.
24. Mills SE. The Breast. In:Carter D, Schnitt SJ, Mills RR, editors. Stenberg's Diagnostic Surgical Pathology. $5^{\text {th }}$ ed. Baltimore, Philadelphia: Lippincott Williams \& Wilkins; 2010; p285-350

25. Ye X, Lu D. HER2 and VEGF expression in breast cancer and their correlations. Chin.-Ger. J. Clin. Oncol. 2010 April; 9(4):208-12. doi:10.1007/s10330010-0018-6

26. Esraa A. Dujaily AL, Al-Kelabi LH, Alkhafaji AJN, Al-Harris ESM. HER-2/neu overexpression in correlation to Vascular Endothelial Growth Factor, grade and stage of Non other wised specified Invasive ductal carcinoma. Al-Qadisiah Medical Journal. 2010; 6 (10): 27-37

27. Semenza, GL. HIF-1: Using two Hands to Flip the Angiogenic Switch. Cancer Metastasis Rev. 2000 June;19(1-2):59-65. doi:10.1023/A:1026544214667.

28. Blackwell KL, Dewhirst MW, Liotcheva V, Snyder S, Broadwater G, Bentley R, Lal A, Riggins G, Anderson S, Vredenburgh J, Proia A, Harris LN. HER2 gene amplification correlates with higher levels of angiogenesis and lower levels of hypoxia in primary breast tumors. Clin Cancer Res. 2004 Jun 15;10 (12 Pt 1) :4083-88.

\section{How to cite this article?}

Sahu N, Sharma A, Senapati U. HER-2/neu overexpression correlates with increased expression of VEGF in primary breast carcinoma. Int J Med Res Rev 2016;4(12):2092-2099.doi:10.17511 /ijmrr. 2016.i12.03. 